\title{
Cusp overlap technique for transcatheter self-expanding aortic valve implantation
}

\author{
Jerzy Sacha ${ }^{1,2}$, Krzysztof Krawczyk ${ }^{1}$, Witold Gwóźdź ${ }^{3}$, Jarosław Bugajski ${ }^{1}$, Mariusz Darmetko³, Marek Gierlotka ${ }^{1}$ \\ ${ }^{1}$ Department of Cardiology, University Hospital, Institute of Medical Sciences, University of Opole, Opole, Poland \\ ${ }^{2}$ Faculty of Physical Education and Physiotherapy, Opole University of Technology, Opole, Poland \\ ${ }^{3}$ Department of Cardiac Surgery, University Hospital, Institute of Medical Sciences, University of Opole, Opole, Poland
}

Adv Interv Cardiol 2021; 17, 2 (64): 230-231

DOI: https://doi.org/10.5114/aic.2021.107509

Transcatheter aortic valve implantation (TAVI) may cause atrioventricular conduction disturbances and, consequently, the necessity for permanent pacemaker implantation (PPI). This is because the His bundle surfaces at the basal part of the membranous septum (in the neighbourhood of the non-coronary cusp) and implant depth greater than the length of the membranous septum may interfere with the conduction system [1]. To mitigate this risk, the valve should be implanted at a high position to minimize the contact of the valve frame with the base of membranous septum. During TAVI, the valve is usually positioned in a coplanar fluoroscopic projection with 3 cusps view, but for a self-expanding valve, like the Evolut R (Medtronic, Minneapolis, Minnesota), such a view does not ensure a high implantation. However, a recently proposed technique based on a cusp overlap allows valve implantation at a very high position and significantly reduces the risk of PPI [2, 3].

In this technique, the coplanar projection with the right and left cusps overlap is determined in multi-slice computed tomography, and such a projection is used for the valve implantation. Conversely to the standard 3-cusps view, which is based on a left-anterior oblique projection, the cusp overlap requires a right-anterior oblique (and usually caudal) projection. In such an overlap view, the lowest point of the noncoronary cusp is perfectly exposed (Figure $1 \mathrm{~A}$ ), and hence the valve may be positioned extremely high without a risk of pop-out.
During the implantation, the valve should be initially positioned above the annulus, with its marker band at the level of half of the pig-tail tip (Figure $1 \mathrm{~B}$ ). Then, while pacing at a rate of around $140 \mathrm{bpm}$, one should start releasing the valve - in such an approach, the valve spontaneously steps down towards the left ventricle and often reaches a proper final position by itself (Figure $1 \mathrm{C}$ ). It is worth noting that in a cusp overlap view the valve frame parallax is usually removed. Next, one must change the projection to the coplanar 3-cusp view in order to confirm the proper valve relation to the left cusp (Figure $1 \mathrm{D}$ ). Before releasing the valve, the stiff guidewire should be pulled back to remove any tension within the delivery system, and the release must be carried out very slowly (for approximately $30 \mathrm{~s}$ ) without moving the delivery system, to avoid valve pop-out.

It should be emphasized that a very high valve implantation position is usually unachievable in the coplanar 3-cusp view (Figure 1 D) because the operator will not accept the risk of valve pop-out. In a recent study, the presented cusp overlap technique reduced the risk of PPI after TAVI with self-expanding bioprosthesis below 5\% [3]. The described technique is straightforward, safe, and may be easily implemented by TAVI operators.

\section{Conflict of interest}

The authors declare no conflict of interest. 

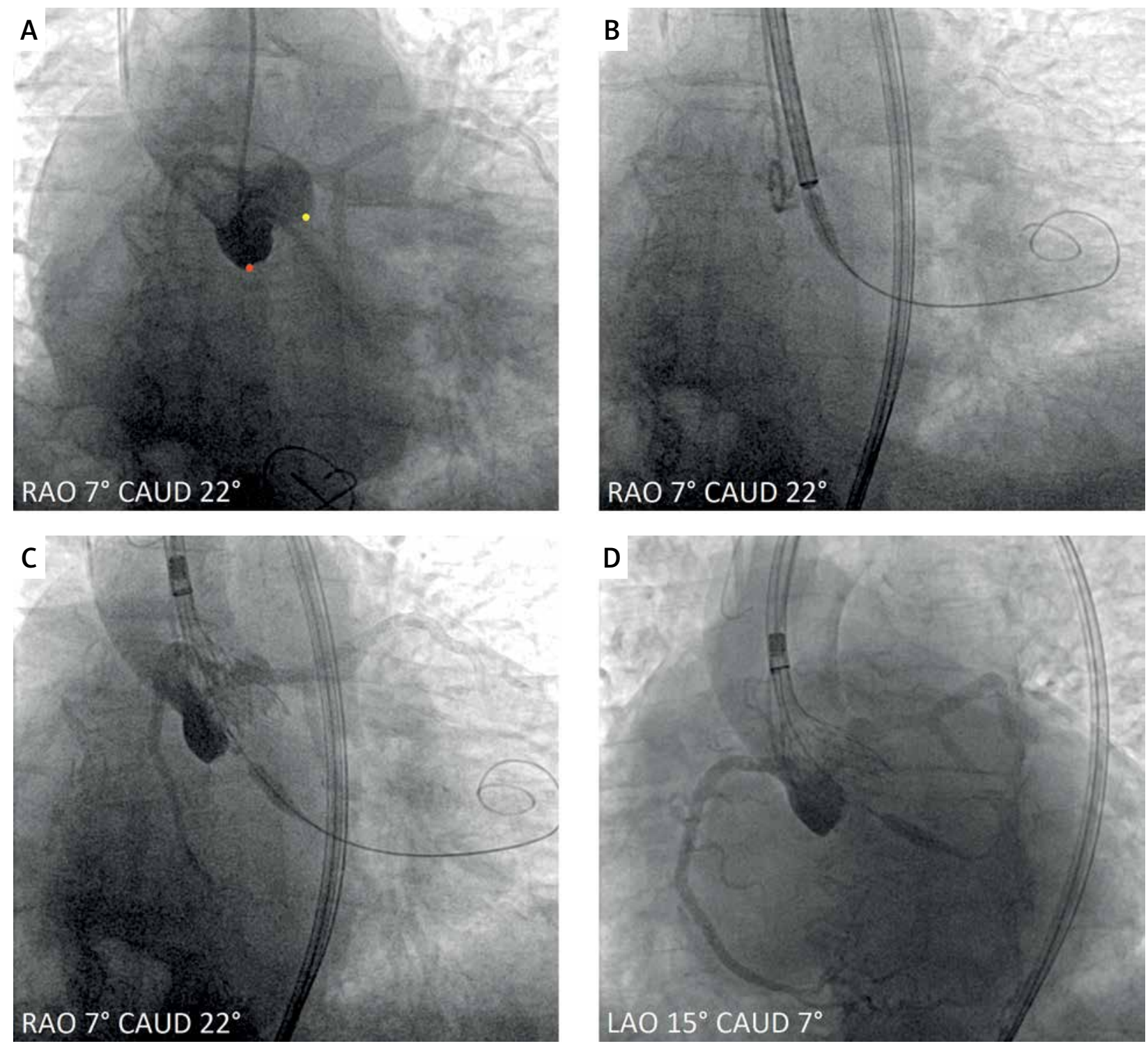

Figure 1. The Cusp overlap technique. A - Aortography in the coplanar projection with the right and left cusps overlap (RAO $7^{\circ}$ CAUD $22^{\circ}$ ). The pig-tail is sitting in the noncoronary cusp (the hinge point of the cusp is marked with a red dot), but the right and left cusps overlap (the overlap hinge points are marked with yellow dots). This projection is also ideal for crossing the native valve because it separates the noncoronary cusp from the right and left ones. B - The initial position for the valve release: the marker band is located at half the height of the pig-tail tip. $\mathbf{C}$ - While releasing, the valve spontaneously goes down to the left ventricle and most often reaches the proper position by itself - during this procedure the heart should be paced at a rate of about $140 \mathrm{bpm}$ (in this case, pacing was carried out using a Confida stiff guidewire). D - The coplanar 3-cusp view (LAO $15^{\circ}$ CAUD $7^{\circ}$ ) shows the valve position in relation to the left and noncoronary cusps. This is the final valve position in which it was released - to remove any tension within a delivery system and prevent valve pop-out, the stiff guidewire was retracted CAUD - caudal, RAO - right anterior oblique, LAO - left anterior oblique.

\section{References}

1. Jilaihawi H, Zhao Z, Du R, et al. Minimizing permanent pacemaker following repositionable self-expanding transcatheter aortic valve replacement. JACC Cardiovasc Interv 2019; 12: 1796-807.

2. Tang GHL, Zaid S, Michev I, et al. "Cusp-overlap" view simplifies fluoroscopy-guided implantation of self-expanding valve in transcatheter aortic valve replacement. JACC Cardiovasc Interv 2018; 11: 1663-5.
3. Pisaniello AD, Makki HBE, Jahangeer S, et al. Low rates of permanent pacing are observed following self-expanding transcatheter aortic valve replacement using an annular plane projection for deployment. Circ Cardiovasc Interv 2021; 14: e009258. 